\title{
Remodelling of specialist services enables safe reduction in hospital admissions of patients with sickle cell disease: Lessons from the COVID-19 pandemic
}

Sickle cell disease is characterised by recurrent painful crises often leading to hospitalisation. During the COVID-19 pandemic, it was important to try to reduce the need for hospital admission for these high-risk patients while at the same time ensuring that hospital avoidance did not put them at risk of deterioration from disease-related complications. In the 3-month period between March and May 2020, there was a significant reduction in the number of hospital admissions as well as mean length of stay compared with the mean figures over the same months in the preceding 5 years (2015-19), with an overall reduction in inpatient days of $77 \%$. There were no cases of unsafe hospital avoidance or presentations to hospital that were inappropriately delayed. Frequent telephone communication with patients and provision of ambulatory care were, among others, two very important means of supporting our patient population.

KEYWORDS: Sickle cell disease, vaso-occlusive crisis, COVID-19

DOI: 10.7861/clinmed.2020-0474

\section{Background}

In England, sickle cell disease (SCD) affects about 1 in 2,000 live births and around 12,500-15,000 individuals are currently estimated to be living with SCD. It is one of the most common

Authors: ${ }^{A}$ consultant haematologist and clinical lead for haemoglobinopathies, Homerton University Hospital NHS Foundation Trust, London, UK; Blead nurse for haemoglobinopathies, Homerton University Hospital NHS Foundation Trust, London, UK; 'ST5 haematology, Homerton University Hospital NHS Foundation Trust, London, UK; DFY2 haematology, Homerton University Hospital NHS Foundation Trust, London, UK; ${ }^{E}$ Clinical psychologist, Homerton University Hospital NHS Foundation Trust, London, UK; ' F consultant haematologist, Homerton University Hospital NHS Foundation Trust, London, UK; ' 'service manager, Homerton University Hospital NHS Foundation Trust, London, UK; ${ }^{H}$ GP and clinical lead for haemoglobinopathies, The Hoxton Surgery, London, UK and NHS City and Hackney Clinical Commissioning Group, London, UK; Ibiomedical scientist, The Scientists Laboratory, London, UK single gene disorders in the UK. ${ }^{\text {SCD }}$ is caused by a single amino acid substitution resulting in the production of sickle haemoglobin ( $\mathrm{HbS}){ }^{2} \mathrm{HbS}$ polymerises when deoxygenated, resulting in red-cell sickling which in turn leads to vaso-occlusion and haemolysis. Painful vaso-occlusive crises (VOCs) constitute the hallmark of SCD and are by far the most common cause of hospital admission for these patients. They are unpredictable and recurrent, leading to poor quality of life and high hospital utilisation. ${ }^{3}$ Our institution is a specialist centre for SCD, with a caseload in excess of 350 patients.

\section{The challenge}

At the beginning of March 2020 and as cases of COVID-19 were rising alarmingly, there was increasing concern and a pressing need to institute measures for our SCD population, who are considered 'high risk' due to their impaired immunity resulting from functional hyposplenism, systemic vasculopathy that predisposes them to end organ dysfunction and their high risk of thrombosis. ${ }^{4}$

Disseminating information and trying to reduce the need for hospital attendance while ensuring that hospital avoidance does not put patients at risk from other complications of their disease was our top priority.

\section{Our strategy}

In the first instance, we issued a letter with details about COVID-19 and protective measures, as well as instructions for patients who may be concerned about having been infected. This was largely based on public health guidance, but special emphasis was put on instructing patients with fever not just to self-isolate due to their high risk of bacterial sepsis, and contact details of our service for advice were provided. This letter was sent to individual patients and was also posted on our website ${ }^{5}$ and social media.

We also felt that preserving the normal functions of the service for as long as possible was very important in reducing the need for acute hospital admissions, and with the support of our management team this was achieved.

Our day unit normally serves as a walk-in centre for ambulatory care of SCD patients in crisis during working hours. This facility continued to operate during the pandemic, as a means of alleviating pressure from the emergency department (ED) and reducing the need for hospital admission. In fact, its function was further enhanced by making it available on bank holidays as well. The day unit was restructured to ensure social distancing. A 
large number of patients with SCD are on a regular transfusion programme for prevention of complications including recurrent VOCs. This was also allowed to continue, as any interruption could lead to patients becoming more symptomatic. The situation was continually reviewed and contingency plans for all aspects of the service were in place for a stepwise reduction of activity if new circumstances arose, such as staff redeployment or blood shortages.

Unlike most outpatient clinics in the hospital that were greatly reduced or cancelled, our two SCD clinics per week continued as usual, but over the phone. In addition, apart from routine telephone consultations with patients as part of their scheduled clinic appointments, a large number of patients, especially the more severely affected, were regularly contacted by phone to ensure that they were well while at the same time emphasising the need to present to hospital if they became ill. The latter was crucial to ensure that patients were not avoiding presenting to hospital, risking deterioration at home and delayed presentation for treatment. Patients were also reminded of scheduled treatments such as transfusions and the need to attend, while other issues such as their supply of medicines and the need to comply with social distancing rules were also addressed. At the same time, the clinical psychologist of our team was also in contact with her regular clients as well as patients flagged to her by the clinica team as potentially needing her input and support.

This constant and ongoing communication was greatly appreciated by patients and was reassuring to the treating team that patients remained safe in the community. There were two very encouraging messages that became apparent through these communications: firstly, the patients engaged much more with their GPs than they had done previously, and secondly, a substantial number of patients with a history of very frequent hospital admissions for pain management were realising that they were not feeling any worse than before and often described themselves as feeling 'better' and 'stronger'. There were no cases where we felt concerned about unsafe hospital avoidance and no presentations to hospital that were inappropriately delayed. In addition, we have avoided prescribing more opiate analgesia to use at home, in order to avoid potentially delayed presentation to hospital when this was absolutely necessary.

The number of hospital admissions in March 2020 was 40, compared with a mean of 57 in March over the preceding 5 years (2015-19), representing a 30\% reduction. In April 2020 there were 21 admissions compared with a mean of 52 in the same month in 2015-19, representing a 60\% reduction, and in May, 24 compared with 54 in 2015-19, a 56\% reduction (Fig 1a). Equally, the mean length of stay for March, April and May 2020 was 2.3, 3.0 and 2.4 days compared with a mean of 5.0, 5.4 and 5.5 days between 2015 and 2019 respectively (Fig 1b). Overall, the total mean number of inpatient days for management of uncomplicated VOCs from March to May 2015-19 was 822, while over the same period in 2020 it was 189, representing a 77\% reduction (Fig 1c).

We have also analysed data for the 10 patients in our service with the highest hospital attendance rate. Between 2015 and 2019 , the mean number (range) of inpatient days per month for pain management of this group of patients was 10.6 (3.5-14.6). The relevant figure in the 3-month period between March and May 2020 was 3.3 (0-12.7) days per month, a 66\% reduction (Fig 1d).

\section{The day after}

There is great uncertainty as to how long COVID-19 will continue to pose a threat for our SCD population. Besides that, hospitalisation
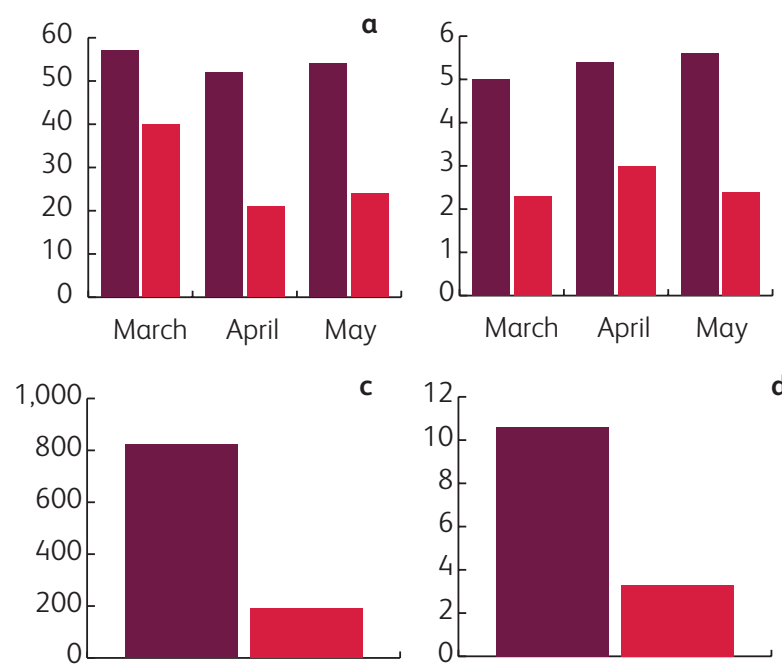

Fig 1. Hospital admissions for vaso-occlusive crisis in March-May 2015-19 (dark red) and 2020 (red). a) Mean number of admissions. b) Mean length of stay. c) Total inpatient days. d) Hospital days per month for the 10 most frequently attending patients.

always carries many risks such as hospital-acquired infections and thrombosis, and leads to increased overall morbidity. ${ }^{6}$ The great reduction in hospitalisation for VOCs that we saw between March and May 2020 was largely the result of the patients' anxiety about contracting COVID-19 and was further supported by the reconfiguration of our services. The COVID-19 outbreak may thus have inadvertently provided a valuable experience for both patients and service providers that could potentially help the SCD population reduce the rate of hospital admissions in a safe manner even after the pandemic has subsided.

Sharing the experience of these 3 months with our patients, paired with education around the risks associated with hospitalisation, is the first step. Showing our patients what they collectively achieved, their ability to self-manage safely with the appropriate support and the reduced hospitalisation required can lead to patient empowerment. With that in mind, we are starting an online campaign in collaboration with the patient-run support group. We have already created and released a YouTube video as a means of communicating rapidly and with the largest possible audience, sharing this experience with our patients. ${ }^{7}$ This has been widely viewed and very well received, with many positive comments posted online and many more sent by text messages to our clinical nurse specialists. Given its success, we will be using this platform for rapid communication with patients regarding changes in services, health information or educational material.

We have also conducted a patient satisfaction survey, receiving 78 responses which represents over $20 \%$ of our caseload. $77 \%$ of patients agreed $(35 \%)$ or strongly agreed $(42 \%)$ with positive statements about our services during the COVID-19 pandemic (questions 1-5, Table 1). In the same survey, it was clear that the vast majority of patients felt that they understood the Department of Health guidance regarding shielding and were compliant with it (questions a-b, Table 1).

Widely available robust ambulatory care is an intervention that we also found to be highly effective. Despite the dramatic reduction in hospital admissions, the activity of our day unit was 
Table 1. Patient satisfaction survey of services during the COVID-19 pandemic

During the COVID-19 pandemic ...

1. I had adequate information from the sickle cell and thalassaemia team about how COVID-19 might affect me

2. I felt my sickle cell / thalassaemia care continued without interruption

3. I knew how to contact the sickle cell and thalassaemia team if I was concerned

4. I felt well supported by the sickle cell and thalassaemia team

5. I found telephone consultations and clinics were addressing my health issues adequately

\section{Cumulative}

a. I have understood the Department of Health guidance about 'shielding'

b. I have followed the Department of Health guidance about 'shielding'

$\mathrm{A}=$ agree; $\mathrm{D}=$ disagree; $\mathrm{NN}=$ neither agree nor disagree; $\mathrm{SA}=$ strongly agree; $\mathrm{SD}=$ strongly disagree reduced only slightly, if at all. We are currently considering making it available during weekends as well.

We found telephone clinics and unscheduled telephone communications very effective and well received by patients, so we intend to establish those in a structured manner on a permanent basis. Face-to-face consultations will continue, but will greatly reduce in numbers and be complemented by frequent telephone clinics. We are working with our trust to establish robust measures to ensure data protection and identification of patients. As telephone clinics are less resource demanding and more convenient for patients, they can increase the overall frequency at which patients are assessed and allow closer monitoring, especially of the more complex patients. Such remote consultations can be further improved by using FaceTime or Skype. Such consultations will have the same format and will be supervised in the same manner as standard face-to-face appointments, so we do not anticipate any compromise in the training offered to specialist trainees. All patients will still have a face-to-face annual review and, for a very small number such as those with limited access to technology or those with hearing problems or learning difficulties, face-to-face consultations will remain the only means of reviewing them.

In the same context, there is a unique opportunity to further enhance the role of primary care and community services, and to improve the historically poor patient engagement in order to have a more comprehensive and multi-layered approach to the management of this chronic illness. In our geographical area, we have already established shared care pathways with local GPs ${ }^{8}$ which we intend to utilise further, while a similar drive is seen in primary care, with the majority of consultations being videoassisted or by telephone and there is a current pilot to see how some of the learning from the pandemic and a more 'digital' approach can be maintained.

Specimens such as blood or urine can be collected in the community, and with that in mind we are creating a satellite phlebotomy facility in our community SCD and Thalassaemia Centre. Alternatively, telepathology services can be utilised to enable patients to take their own samples when required and send to the laboratory by post. Already available technologies can also be explored; examples include electronic pain or other symptom diaries and real-time continuous recording of biological parameters through wearable devices, with platforms built for data to be downloaded and made available to the clinical teams.
The ongoing crisis has demanded unconventional and innovative thinking from all healthcare professionals. We believe that there are many lessons to be learnt that can improve the chronic management of patients with SCD in the future, leading to patient empowerment and reducing disruption of their daily lives, while ensuring, even enhancing, the quality of their care.

\section{Acknowledgements}

Special thanks to Nikolas Tsitsikas for his technical support in creating the video for the patients of our service.

\section{References}

1 Sickle Cell Society. Standards for the clinical care of adults with sickle cell disease in the UK, second edition. London: Sickle Cell Society, 2018. www.sicklecellsociety.org/wp-content/ uploads/2018/05/Standards-for-the-Clinical-Care-of-Adults-withSickle-Cell-in-the-UK-2018.pdf [Accessed 27 August 2020].

2 Kato GJ, Piel FB, Reid CD et al. Sickle cell disease. Nat Rev Dis Primers 2018:4:18010

3 Platt OS, Thorington BD, Brambilla D] et al. Pain in sickle cell disease. Rates and risk factors. N Engl J Med 1991;325:11-6.

4 Ware RE, de Montalembert M, Tshilolo L, Abboud MR. Sickle cell disease. Lancet 2017;390:311-23.

5 Homerton University Hospital NHS Foundation Trust. Sickle cell service \& Thalassaemia Service. www.homerton.nhs.uk/sickle-cellservice-thalassaemia-service [Accessed 27 August 2020].

6 NHS Digital. Summary Hospital-level Mortality Indicator (SHMI) Deaths associated with hospitalisation, England, April 2019 - March 2020. https://digital.nhs.uk/data-and-information/publications/ clinical-indicators/shmi/current [Accessed 27 August 2020].

7 Tsitsikas DA. Homerton sickle legends. https://www.youtube.com/ watch? $v=c t X W 53 i w m x Y$ [Accessed 27 August 2020].

8 Homerton University Hospital NHS Foundation Trust and City and Hackney CCG. City and Hackney adult referral pathway for sickle cell disease (SCD) and thalassaemia. https://gps.cityandhackneyccg. nhs.uk/cdn/serve/pathway-downloads/1539364493-af3d441f7776d1aff454679656158782.pdf [Accessed 27 August 2020].

Address for correspondence: Dr Dimitris A Tsitsikas, Homerton University Hospital NHS Foundation Trust, Homerton Row, Clapton, London E9 6SR, UK.

Email:dimitris.tsitsikas@nhs.net 